\title{
NETWORK FAULT DIAGNOSIS USING DATA MINING CLASSIFIERS
}

\author{
Eleni Rozaki \\ Cardiff School of Computer Science \& Informatics, \\ Cardiff University, Cardiff, UK \\ E.Rozaki@cs.cardiff.ac.uk
}

\begin{abstract}
Mobile networks are under more pressure than ever before because of the increasing number of smartphone users and the number of people relying on mobile data networks. With larger numbers of users, the issue of service quality has become more important for network operators. Identifying faults in mobile networks that reduce the quality of service must be found within minutes so that problems can be addressed and networks returned to optimised performance. In this paper, a method of automated fault diagnosis is presented using decision trees, rules and Bayesian classifiers for visualization of network faults. Using data mining techniques the model classifies optimisation criteria based on the key performance indicators metrics to identify network faults supporting the most efficient optimisation decisions. The goal is to help wireless providers to localize the key performance indicator alarms and determine which Quality of Service factors should be addressed first and at which locations.
\end{abstract}

\section{KEYWORDS}

Fault diagnosis, Weka classifiers, Rules, Decision trees, Bayesian networks.

\section{INTRODUCTION}

In this paper, we propose and develop an automated scheme for monitoring and fault detection based on data mining techniques to provide converged services with functionalities that facilitate its fault-handling and operational management. With the use of Weka classifiers (rules, trees, Bayes), one of the main challenges is to carry out effective and accurate monitoring of services with the detection and classification of possible faults that may arise at runtime so that appropriate measures rules and decision trees can be made to ensure correct fault-handling and compliance with the Quality of Service (QoS) constraints established between service providers and end-users [1].

The FCAPS framework was defined and implemented in 1997 as a management framework for network provides. The FCAPS framework stands for fault, configuration, accounting, performance, and security. Within this framework, performance management is the process of quantifying, measuring, analysing, and controlling the performance of the components of a network. Fault management is the process of acquiring, revealing, and then counteracting faults that exist in the network. Configuration management is the process of obtaining and controlling the configuration parameters of a network. Accounting management refers to usage statistics, cost allocation, and pricing. Security management is the process of monitoring access to a network based on defined policies [2].

Jan Zizka et al. (Eds) : DBDM, CICS, CCNET, CSIP - 2015

pp. 29-40, 2015. (C) CS \& IT-CSCP 2015

DOI : $10.5121 /$ csit.2015.50703 
We are working in the diagnosis stage and investigating how to automate the process of setting up a fault classification algorithm based on KPI alarms to identify network faults. At the same time, we investigate classification rules and decision tree algorithms between different alarms of KPIs based on causes and symptoms of network malfunctions and demonstrate both system performance and localisation of the KPI alarms. [3].

Within the academic literature, a great deal of time and effort has been devoted to methods of fault management and fault diagnosis within wireless networks. A variety of methods, such as hierarchical support vector machine, the creation of assessment indexes, and neural algorithms based on faculty connectors [3][4][5].

There has been a limited amount of research regarding the degree of fault alarm under data mining techniques. This is problematic given that defect management on telecommunication networks has been studied extensively utilizing the correlation techniques. In this way, the current paper helps to move forward the existing knowledge regarding fault diagnosis.

\section{Data Mining Process}

The process of mining data so that it can be gathered and used to make decisions does not involve a single activity. Instead, data mining involves a series of steps that must be undertaken in order to allow large amounts of data to be reduced and put into a format that can be used for the purpose of decision-making [7].

The first step in the data mining process is known as data cleaning. In this step, data are examined to determine which data are relevant for the given task or purpose. Irrelevant data and data that may be considered to be invalid are removed. Next data integration occurs in which data from multiple sources are combined into a single source or dataset. It is often the case that the data that are combined are heterogeneous [8].

The next step in the data mining process is data selection in which further examination of the data occurs in order to determine which data should be taken from the dataset to be used for the task at hand. Once the data selection has been completed, then data transformation can occur. This stage in the data mining process involves transforming the selected data into appropriate formats for the mining procedure. [8].

The next step in the process is the actual data mining. In this step, various techniques for analysing data are used. Then, pattern evaluation is undertaken in which interesting or unique patterns in the data are identified. The identification of patterns occurs in relation to the larger issue or problem that is attempting to be addressed [8].

With the use of Weka, the final step in the data mining process is knowledge representation. This process of incorporating Weka tools into the data process requires analysing and pre-processing the data, as well as examining the validity of the data. Then, class attributes must be defined followed by the actual extraction of the features that will be used for classification. Next, a subset of features is selected to be used as part of the knowledge construction [8].

The next part of the knowledge representation using Weka is the investigation of any imbalances in the selected data and a determination of how those imbalances may be counteracted. Then, a subset of instances is chosen. The subset of instances might be the records upon which the learning will be based. Following the selection of the subset of instances, the classifier algorithm is applied for the learning process. Finally, a testing method is chosen in order to estimate the performance of the algorithm [8]. 


\section{DATA MINING TECHNIQUES}

There are a large number of tools that can be used in data mining to handle a variety of tasks, such as data pre-processing, regression, clustering, feature selection, and even visualization. However, it is important to understand that the various tasks that can be performed with data mining tools require different algorithms. At the same time, a programming environment is needed to run the algorithms [9]. In this study, the Waikato Environment for Knowledge analysis (Weka) has been chosen because of its ability to run a variety of different algorithms and its general robustness [10].

An important part of this study is understanding the classifiers that are used for the network faults detection. The classifiers that are of importance to this study are briefly explained.

\section{1. $\mathrm{J48}$ tree}

The J48 tree functions through the creation of decision trees in which data are examined with the goal of reducing the data into small subsets under the smallest subset can be found at which point an optimised outcome has been determined. A leaf node is created within the decision tree to indicate that a particular class should be chosen [9][11].

\subsection{LAD Tree}

The Logical Analysis of Data (LAD) tree works by creating a classifier for a binary target. The classifier is created through a process in which a logical expression is learned that has the ability to determine positive and negative samples in a dataset. The underlying theory of the LAD tree is that any binary point that contain some positive patterns but not negative patterns could be classified as positive. At the same time, any binary point that has negative patterns but no positive patterns can be classified as negative. In a similar manner to the J48 tree, a large set of patterns occurs and then subsets are determined until a subset is determined that satisfies the patterns in the data [11].

\subsection{JRip}

The JRip classifier, which was introduced in 1995 by W. W. Cohen, is an optimised version of an older classifier that Cohen created [12]. JRip was implemented with the prepositional rule learner known as Repeated Incremental Pruning to Produce Error Reduction (RIPPER), meaning that it has the ability to replace or revise rules. With JRip, it is possible to isolate some of the data being examined for training. At the same time, a decision can be made regarding the set of rules generated for selected attributes.

\subsection{PART}

The PART algorithm [24] is an algorithm that does not function to generate accurate rules through global optimisation. Instead, PART functions to build a rule and then remove the instances that it covers in order to create a recursive rule until there are no instances that remain. Another way of thinking about the PART algorithm is that creates decision lists. With the decision lists, new data are compared to the rules in the decision list. Upon the first match of a rule, data are assigned to that rule. In this way, PART makes the best leaf into a rule rather than the optimised leaf [9]. 


\subsection{Naïve Bayes}

The Naïve Bayes classifier [17] operates in accordance with the Bayes rule of conditional probability. The classifier uses all of the attributes of a dataset by analysing them individually. The idea is that all of the attributes are equally important. No single attribute is more important than another attribute. At the same time, all of the attributes of the data are considered to be independent of each other [8].

\subsection{Bayes Networks}

Bayesian networks, or Bayesnet, are also known as belief probabilistic networks. It is the structure of a Bayesian network that determines the relationships and dependencies between the variables in a dataset. Because of the fact that the structure of Bayesian networks determine dependencies and relationships between variables, the use of such networks have been proposed for use in the diagnosis of faults in some cellular networks [13].

\section{DATA PREPARATION}

The work of data preparation begins with analysing and pre-processing of database features, as well as an assessment of the correctness of the data that are to be analysed. The work of data preparation involves determining the specific data that can be used to identify network malfunctions. The selection of the data to be analysed involves several considerations, such as which data are representative of the KPIs (symptoms), data availability (inputs), the attributes of the data, and establishing limits of the network faults [9][12]. The next important issue is the generation of the alarms, as well as the relationship between the KPIs and alarms. Before discussing alarms, it is appropriate to briefly explain the difference between an alarm and a fault. An alarm is an indication of a problem or issue in a network. An alarm can be thought of as the outward symptom of a problem. In contrast, a fault is the actual cause of a problem in a network.

The fault is the actual issue that needs to be corrected in order to restore a network to optimisation [2]. The alarms are generated when the nodes of the KPIs cross a specific limit or threshold that is pre-determined. It must be recognized that the thresholds are not set randomly. Instead, the thresholds are set based on their ability to provide an indication of a problem. At the same time, the threshold levels must be such that the probability of false alarms is minimised [2].

After each KPI is associated with an alarm each alarm is associated with a specific security level. An example might be a binary alarm that has the possible conditions or states or On and Off. If the KPI should be On, but it is found to be Off, then an alarm occurs. A KPI can have as many states or conditions as necessary. Some KPIs might have three states, while others might have four or even five states [2].

The KPIs are derived with the help of counters using different formulations. A single counter helps to provide a very limited indication of the larger network. However, with the use of several counters, it is possible to gain a much broader view of the network. In this study, the evaluation is presented on the basis of four major KPIs used as an input to create a classification algorithm to perform the data for the pre-processing process [13]. Table 1 shows the four KPIs that are used are Call set up success rate (CSSR), Call Drop Rate (CDR), Handover Failures (HOF) and Call Traffic Rate (TR). In addition, each KPI can take one of three states: Normal, Critical, or Warning. 
Table 1. KPI alarm metrics

\begin{tabular}{cccc}
\hline \hline Symptom & \multicolumn{3}{c}{ State alarm indicator } \\
\hline \hline KPI & $\begin{array}{c}\text { Normal } \\
(\text { NORM })\end{array}$ & $\begin{array}{c}\text { Critical } \\
(\text { CR })\end{array}$ & $\begin{array}{c}\text { Warning } \\
(\text { WARN })\end{array}$ \\
\hline \hline DCR & DCR $<2$ & $4>\mathrm{DCR}>=2$ & DCR $>=4$ \\
\hline \hline CSSR & $\mathrm{CSSR}>=98 \%$ & $90 \%>\mathrm{CSSR}=>98 \%$ & $\mathrm{CSSR}<=90 \%$ \\
\hline TR & $\mathrm{TR}<60 \%$ & $60 \%>\mathrm{TR}>=70 \%$ & $\mathrm{TR}>=70 \%$ \\
\hline HOF & $\mathrm{TR}<10 \%$ & $10>\mathrm{HOF}>15$ & $\mathrm{HOF}>=15$ \\
\hline \hline
\end{tabular}

Table 1 also shows the specific metrics that have been established as thresholds for each alarm.

The KPI metrics of Handover Failure (HOF) is the ratio of unsuccessful intersystem handovers from $3 \mathrm{G}$ to $2 \mathrm{G}$ over the total number of such attempts. The call dropped rate (CDR) is the ratio of dropped calls over the total successfully established calls [15]. The call set-up success rate (CSSR) is the rate of call attempts until successful assignment. The call drop rate (CDR) is the rate of calls not completed successfully [16]. The traffic rate indicator is the actual rate or percentage of traffic on the network [14].

\subsection{Fault classification algorithm}

We selected the most popular Key Performance Indicators to create a Fault Classification Algorithm. The algorithm parameters must be set up for the input data. The rules generated for the fault classification algorithm are given bellow:

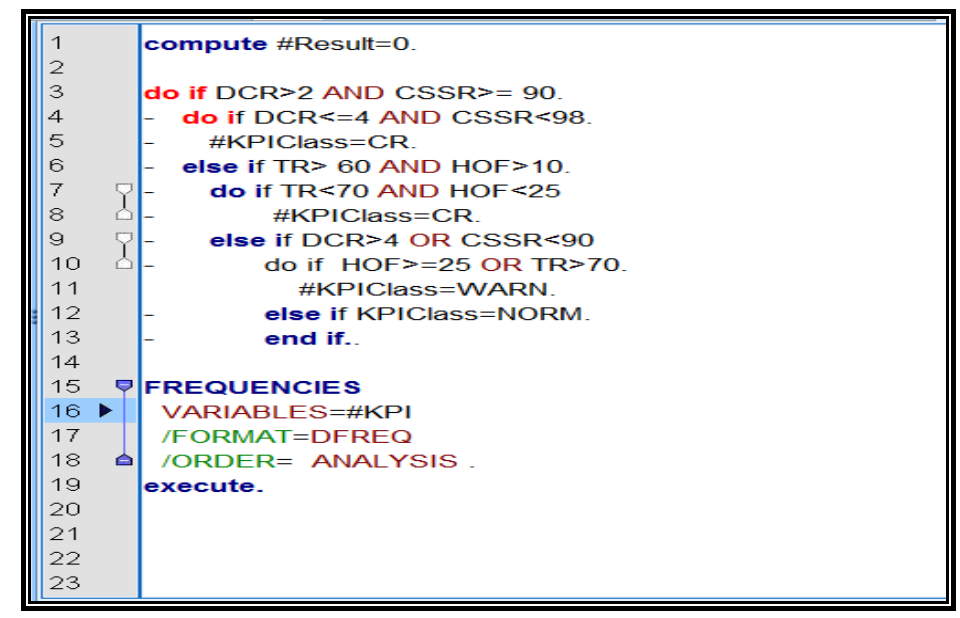

Figure 1. Fault classification algorithm

The parameters will include equality or inequality for the state alarm indicators and greater than or less than for the KPI alarms metrics. The relational nodes can be placed into decisions trees or into rules. However, plans that use relations are more often placed in rules rather than in decision trees. 


\subsection{Explanation of Output Variables}

The next step in the process is the selection of a subset of instances in Weka for the parameters of KPIs. It is important to note that the selection of the subset of instances was not random. Instead, the selection of subsets of instances for this study was based on previous studies and investigations of fault diagnosis in networks using data mining classifiers [14][16][17].

The instance of BSC represents the base station controllers, while Id represents the cell Ids. The Standalone Dedicated Control Channel Congestion Rate (SDCCHCR) indicates the probability of accessing a stand alone dedicated control channel available during call set up [14] [17]. TCH Seizure Attempts is the number of traffic channels that are allocated for traffic [17]. The $(\mathrm{SDCCH}) \mathrm{Access}$ Rate shows the percentage of call access success rate received in the base station location [17]. TCH Availability is the rate of Traffic Channels availability in the network, while the TCH Drop is the total drop rate in the network and also the TCHCR is the rate of TCH congestion in a network area [14]. Finally, the TCHTR is the rate of incoming and outgoing traffic of a channel [16].

The Handover Success parameter shows the percentage of success handovers on the network of all handover attempts [15]. The SDCCH Drops Excessive TA presents the number of drops due to excessive timing advance [17]. Based on those relational nodes, the system would identify the optimised status of the TCH (traffic channel), RAN (radio accessibility network performance audit) and show their optimised states [17]. The Traffic Channel Congestion Rate of the SDCCH drops measures the total number of RF losses as a percentage of the total number of call attempts for the SDCCH channels on the network. The SDCCD Availability Rate is the percentage of SCDDH channels that are available on a network at a given time [14].

The SCDDHD Sudd Lost Con is the rate of connections that are suddenly lost on a network [17]. Additionally the TCH Drop Rate is the rate of traffic assignment failures, while TCHCR is the congestion rate related to call setup traffic [17]. The HOFR statistic is intended to give an indication of the rate as a percentage of handover failures in relation to total handovers [14]17]. The TCHSS shows the percentage of TCHs that are successfully seized [17]. The SDCCH Drops is the number of drops due to low signal strength or network congestion [14] [17]. The Handover Success Rate is the percentage of rate of handover successes. Finally, the KPI Alarms are the symptoms that occur due to the establishing limits that are set by the classifiers of the system.

\section{IMPLEMENTATION OF THE SYSTEM}

The implementation of alarm detection and fault localizations may generate a large number of alarms. The problem with the generation of a large number of alarms is the potential for a high rate of false alarms. The ability for a human to visualize all of the alarms that may be generated is difficult or even impossible. In this way, the alarms generally need to be aggregated so that visualization can occur more easily for the person that is responsible for examining them [2].

Aside from the aggregation of alarms for the purpose of easier visualization, it is also possible to implement an automatic fault identification module that can correlate the observed alarms in order to indicate the root cause of the alarms. With the automatic fault identification module, a human may not even be needed as an automatic recovery system can be used to use the alarms to quickly determine the fault and correct it [2].

For this study, 2,100 instances are inserted that represent the Cell Ids and Base Station locations. In addition, 25 attributes are input that show the data and key performance indicators including 
the data base. The dataset representation in ARFF (Attribute-Relation File Format) is shown below:

@Period,@BSC,@Id,@SDCCHCR,@TCHSeizureAttempts,@TCHAccessRate@TCHAvailabilit y,@TCHDrop,@TCHCR,@TCHTR,@HandoverSuccess,@SDCCHDropsExcessiveTA,@RAN, @SDCCHDR,@SDCCHAvailabilityRate,@HF,@SDCCHDropSuddLostCon,@TCHAssignment FailureRate,@TCHDropRate,@TCHCongestionRate,@HOFR,@TCHSS,@SDCCHDrops,@HO SR@KPIAlarms

Once classification is settled down, representation of data can use data visualization techniques of decision trees and rules classifiers. Figure 2 shows the JRP optimisation rules.

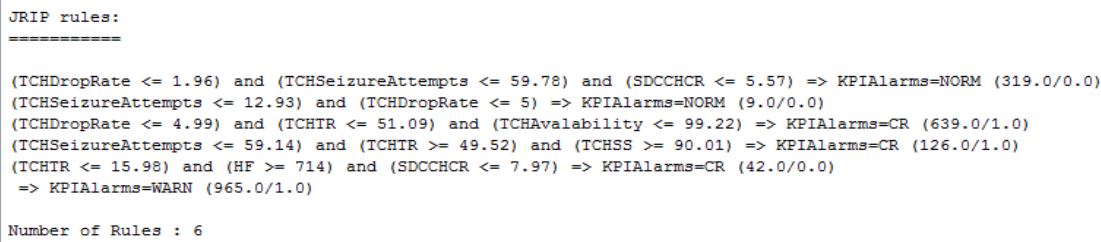

Figure 2. JRP Optimisation rules

The JRP optimisation rules show that six rules were extracted from Weka regarding the JPR classifier. The rules that were generated show the conditions of critical optimization faults and the KPI metrics to show when a cell can be considered to be optimized. Optimization is considered to occur when the Normal (NORM) condition is achieved. The last rule that was generated tells the system that if the cell is not classified as either normal (NORM) or critical (CR), then it needs to be checked for a warning (WARN) status.

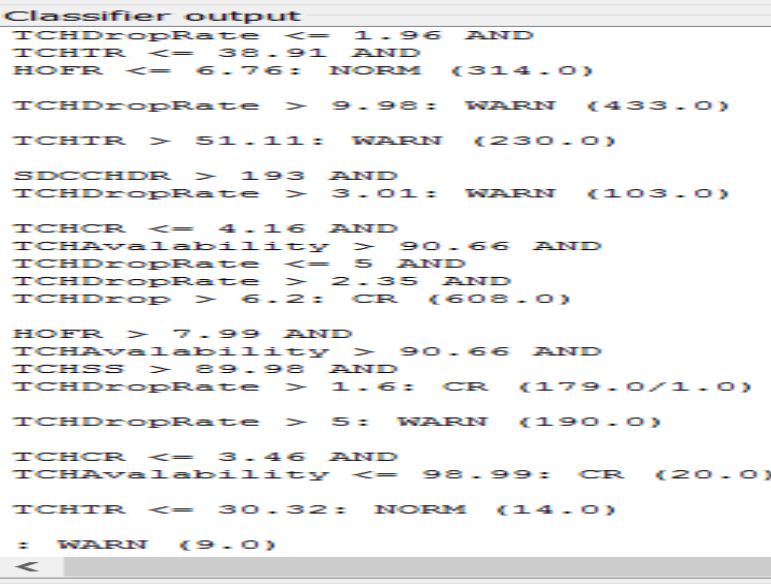

Figure 3. Part optimisation decision list

Figure 3 shows the optimisation decision list. A total of six rules were extracted from Weka regarding the Part classifier. As before, the rules that were generated illustrate the conditions of critical optimization faults and the KPI metrics to show when a cell can be considered to be optimized. Optimization is considered to occur when the Normal (NORM) condition is achieved. The last rule that was generated tells the system that if the cell is not classified as either normal (NORM) or critical (CR), then it needs to be checked for a warning (WARN) status. 


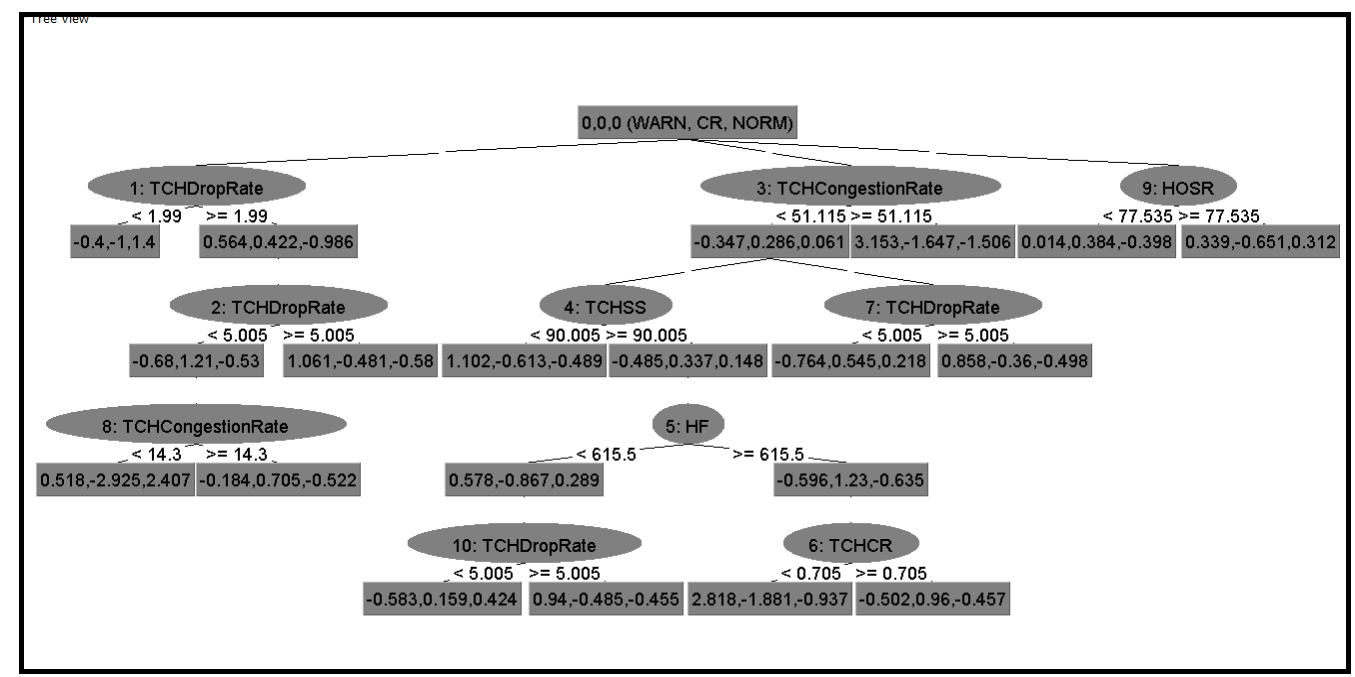

Figure 4. Optimisation decision LADtree

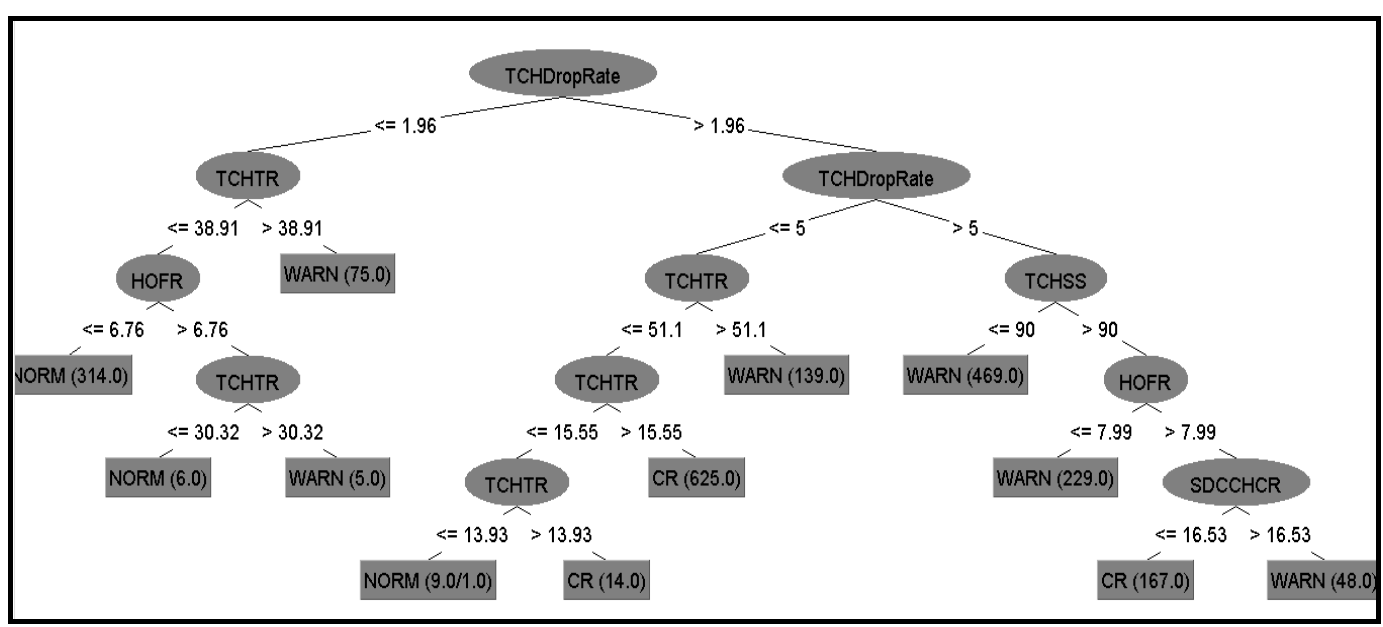

Figure 5. Optimisation decision tree $\mathrm{J} 48$

Figures 4 and 5 show the optimization decision trees for J48 and LAD Tree. The number of Leaves is 12 for J48 class and the Size of the tree is 23 . The attribute selection from the J48 and LADTree classifiers is based on the variables related to the utilization of the network such as Traffic Channels and Handover success and Number. The LAD Tree provides a Logical Analysis of Data as the classification method. The LAD Tree shows a prediction for faults detection that is based on 21 predictor nodes at the tree side and 14 predictor nodes for the optimisation results at the leaves of LAD Tree [11].

Perhaps just as importantly, the decision trees demonstrate the ability to provide a visual representation of the faults that can be understood by a human operator whose job it might be to analyse the data and determine the cause of the lack of network optimisation. By examining the decision trees, it is possible to quickly determine which cells are indicated as being normal (NORM) and which cells are indicated as being critical (CR). By looking at the specific classifiers, the human operator can truly make a quick decision about the fault in the network to correct that fault and return the network to maximum optimisation. 


\section{FAULT DiAgNOSIS RESUltS DisCUSSION}

An average of 860 cells of the data base were classified in a state alarm of "Warning", 785 found to be "Critical" and only 320 cells were considered as "Normal". The most popular KPI metrics extracted by the system are based on TCH Availability, Congestion and Success Rate, Handover Success and Failure Rate, SDCCH and TCH Drop Rate and final TCH attempts.

\subsection{Comparison using different classifiers}

Table II also shows the accuracy alarm prediction by the proposed technique. The table shows that the performance measure results of J48 and PART classifiers have the same performance in terms of accuracy in classification. Both methods achieved an accuracy rate of $99.9 \%$. With the rules and Decision trees classifiers, the accuracy rate is actually more than $99.9 \%$. However, the Bayesian classifiers showed a decline in their accuracy levels as compared to the other methods [12].

This scale by which to measure the performance of algorithms is important in relation to the method that was tested in this study because distributed fault detection and isolation with the use of a Bayes classifier has demonstrated that even if the accuracy is not too high, it is the total output of the use of the classifier that provides important information that can be used by wireless providers to isolate and localize the network malfunctions.

Table 2. Performance measure results

\begin{tabular}{ccccccc}
\hline $\begin{array}{c}\text { Weka } \\
\text { classifiers }\end{array}$ & $\begin{array}{c}\text { Correctl } \\
\mathbf{y} \\
\text { classified } \\
\text { Instances }\end{array}$ & $\begin{array}{c}\text { Incorrectl } \\
\mathbf{y} \\
\text { Classified } \\
\text { Instances }\end{array}$ & $\begin{array}{c}\text { Kappa } \\
\text { Statistics }\end{array}$ & $\begin{array}{c}\text { Mean } \\
\text { absolute } \\
\text { error }\end{array}$ & $\begin{array}{c}\text { Root } \\
\text { mean } \\
\text { squared } \\
\text { error }\end{array}$ & Accuracy \\
\hline \hline Decision Trees & & \\
\hline J48 & 2099 & 1 & 0.99 & 0.0006 & 0.01 & $99.9 \%$ \\
LADTree & 2097 & 3 & 0.99 & 0.0243 & 0.05 & $99.8 \%$ \\
\hline \hline JRIP & 2097 & 3 & 0.99 & 0.0019 & 0.03 & $99.8 \%$ \\
Part & 2099 & 1 & 0.99 & 0.0006 & 0.01 & $99.9 \%$ \\
\hline \hline BayesNet & 1839 & 261 & 0.80 & 0.08 & 0.28 & $87.5 \%$ \\
NaiveBayes & 1701 & 399 & 0.69 & 0.13 & 0.34 & $81 \%$ \\
\hline \hline
\end{tabular}

Table III shows the evaluation of final statistics. The scale is based on the level of accuracy of the algorithm with regards to correctly identifying faults as compared to false results [12]. The final statistics show the accuracy of the model and visualize precision and recall for ROC curve analysis (true positive rate vs false positive rate). [10]. TP rate also demonstrates the sensitivity of the model such as a scale of actual positive values and the FP rate shows the specificity such as the negative tuples that are incorrectly labelled. [11]. In this regard, the slightly less accurate information of BayesNet and NaiveBayes classifiers across the entire system still results in a high 
level of ability to determine the fault or faults that need to be corrected in order to return a network to optimisation [12].

\section{CONCLUSIONS}

This paper proposes a systematic approach for fault diagnosis that is based on a KPI data analysis model using the Weka environment for those who are responsible for the monitoring and optimisation of GSM networks. The importance of the method of fault diagnosis that has been proposed in this paper is that it is one that has not been widely studied. In this regard, a potentially new method, or at least one that has not been greatly considered, is available for network operators who want an automated means by which to quickly identify faults and return networks to optimisation in order to provide a high quality of service to users.

Table 3. Comparison of final statistics weighted avg

\begin{tabular}{lcccccccc}
\hline \hline Classifiers & $\begin{array}{c}\text { TP } \\
\text { Rate }\end{array}$ & $\begin{array}{c}\text { FP } \\
\text { Rate }\end{array}$ & Precision & Recall & $\begin{array}{c}\text { F- } \\
\text { Measure }\end{array}$ & MCC & $\begin{array}{c}\text { ROC } \\
\text { Area }\end{array}$ & $\begin{array}{c}\text { PRC } \\
\text { Area }\end{array}$ \\
\hline \hline J48 & 1.00 & 0.00 & 1.00 & 1.00 & 1.00 & 0.99 & 1.00 & 1.00 \\
LADTree & 0.99 & 0.00 & 0.99 & 0.99 & 0.99 & 0.99 & 0.99 & 0.99 \\
JRIP & 0.99 & 0.00 & 0.99 & 0.99 & 0.99 & 0.99 & 0.99 & 0.99 \\
Ridor & 1.00 & 0.00 & 1.00 & 1.00 & 1.00 & 0.99 & 1.00 & 1.00 \\
BayesNet & 0.87 & 0.034 & 0.913 & 0.876 & 0.884 & 0.833 & 0.993 & 0.98 \\
NaïveBayes & 0.810 & 0.109 & 0.846 & 0.810 & 0.809 & 0.709 & 0.935 & 0.90 \\
\hline \hline
\end{tabular}

The state alarm indicator values shown in the method used in this study provide a strong means by which to perform network diagnosis efficiently and quickly. The rules and decision trees set up that are extracted by the data mining process simplify the optimisation process. The decision trees allow network operates to visually examine multiple network parameters, such as traffic channels and handovers statistics. The end result is that the task performance management network optimisation through fault location finding, and root cause analysis is much easier [6].

It is important to iterate the method of fault diagnosis shown in this paper is not only highly accurate, but it is also efficient. A method of fault diagnosis can be high, but a lack of efficiency in using the method can be ineffective for network operators who must return a network to optimum performance levels in a matter of seconds or minutes, rather than hours [18]. Wireless networks have become more complex with increasing numbers of users [19]. This only adds to the burden of finding automatic methods of fault diagnosis that are efficient, both in terms of accuracy of output and actual usage.

The method of fault diagnosis described in this paper is indeed computationally efficient regarding the rules, decision trees and Bayes classifiers. It also represents optimisation solutions defined by rules. Furthermore, this method of fault diagnosis retains the ability to identify individual KPI metrics and alarm factors severely affecting reliability of fault location calculation. In the end, the results presented in this paper allow for the conclusion that the proposed method has produced very promising results in the classification of multi-class optimisation faults. 


\section{FUTURE WORK}

The results of this study are not only important in terms of what can be concluded from them about the method of network fault diagnosis that was presented, but also the broader context of network fault management. The analysis of network fault diagnosis in this study, and most other studies, requires some knowledge of statistical analysis. The question that arises, however, is whether the people who are responsible for fault diagnosis in practice have the statistical analysis that is needed to efficiently perform the same procedures and analysis conducted in this study and others. While the focus of so much research in this area has been on methods of fault detection, it might be time to study those who have to implement these methods and use them to make realworld decisions.

Another area for future research might be to examine the business decisions that underlie network optimisation efforts on the part of network providers. While the focus of so much of this area of research is on the techniques and methods, the reality is that there are important business decisions that dictate which techniques and methods are used, as well as how they are used [20]. It would be useful to understand the decision-making process and the business realities that determine the specific methods that companies that operate wireless networks use to perform fault diagnosis.

\section{REFERENCES}

[1] D.Adrada, E. Salazar, J. Rojas, and J. C. Corrales, (2014) "Automatic code instrumentation for converged service monitoring and fault detection”, 28th International Conference on Advanced Information Networking and Applications Workshops, pp708-713.

[2] L. Monacelli and R. Francescangeli, (2011) "Fault management for volp applications over wireless and wired ngn networks: an operational prospective", IEEE 36th Conference Local Computer Networks, pp711-718.

[3] A. Rosich, H. Voos, and L. Pan, (2014) "Network design for distributed model-based fault detection and isolation", IEEE Conference on Control Applications, pp1226-1231.

[4] L. Zhang, X. Meng and H. Zhou, (2009) "Network fault diagnosis using hierarchical svms based on kernel method”, 2nd International Workshop on Knowledge Discovery and Data Mining, pp753-756.

[5] R. Ji, J. Gao, G. Xie, G.T. Flowers, and C. Chen, (2014) "A fault diagnosis method of communication connectors in wireless receiver front-end circuits", 60th Holm Conference on Electrical Contacts, pp1-6.

[6] Y-Y. Wang, K-W. Wu, C-M. Huang, and C-C. Chan, (2014) "Quality management and network fault diagnosis for iptv service", 16th Asia-Pacific Network Operations and Management Symposium, pp14.

[7] B. M. Patil, D. Toshniwal, and R. C. Joshi, (2009) "Predicting burn patient survivability using decision tree in weka environment", IEEE International Advance Computing Conference, pp13531356.

[8] P. K. Srimani, and K. Balaji, (2014) "A comparative study of different classifiers on search engine based educational data", International Journal of Conceptions on computing and Information Technology, Vol. 2, pp 6-11.

[9] W. Nor Haizan, W. Mohamed, M. N. M. Salleh, and A. H. Omar, (2012) "A comparative study of reduced error pruning method in decision tree algorithms", IEEE International Conference on Control System, Computing and Engineering, pp392-397. 
[10] I. A. Witten and E. Frank, (2005) Data Mining: Practical Machine Learning Tools and Techniques with Java Implementations, San Francisco, CA: Morgan-Kaufmann.

[11] T. C. Sharma and M. Jain, (2013) "WEKA approach for comparative study of classification algorithm", International Journal of Advanced Research in Computer and Communication Engineering, Vol. 2, pp1925-1931.

[12] W. Shahzad, S. Asad, and M. A. Khan, (2013) "Feature subset selection using association rule mining and JRip classifier", International Journal of Physical Sciences, Vol. 18, pp885-896.

[13] R. Barco, V. Wille, L. Diez, and P. Lazaro, (2006) "Comparison of probabilistic models used for diagnosis in cellular networks”, IEEE 63rd Vehicular Technology Conference, Vol. 2, pp981-985.

[14] M. Panda and S. P. Padhy, (2009) "Traffic analysis and optimization of gsm network", IJSCI International Journal of Computer Science Issues, Vol. 1, pp28-31.

[15] C. Skianis, (2013) "Introducing automated procedures in 3g network planning and optimization", The Journal of Systems and Software, Vol. 86, pp1596-1602.

[16] B. Haider, M. Zafrullah, and M. K. Islam, (2009) "Radio frequency optimization \& QoS evaluation in operational gsm network", Proceedings of the World Congress on Engineering and Computer Science, Vol. 1, pp1-6.

[17] M Ali, A. Shehzad, and M. A. Akram, (2010) "Radio access network audit \& optimization in gsm (radio access network quality improvement techniques)," International Journal of Engineering \& Technology, Vol. 10, pp55-58.

[18] Y. Temprado, C. Garcia, F. J. Molinero, and J. Gomez, (2008) "Knowledge discovery from trouble ticketing reports in a large telecommunications company," International Conference on Computational Intelligence for Modelling Control \& Automation, pp37-42.

[19] R. Barco, L. Diez, V. Wille, and P. Lazaro, (2009) “Automatic diagnosis of mobile communications networks under imprecise parameters”, Expert Systems with Applications, Vol, pp489-500.

[20] V. P. Bresfelean, (2007) “Analysis and predictions on students' behavior using decision trees in weka environment", Proceedings of the ITI 2007 29th International Conference on Information Technology Interfaces, pp51-56.

\section{AUTHOR}

Eleni Rozaki (S-22, M-13) received the M.Sc. degree in Quantitative methods and Informatics from University of Bari Italy in 2008. Currently, she is working toward the PhD degree, in the area of network optimisation at Cardiff University, United Kingdom. Her current research interests include network management, decision support systems and data mining techniques.

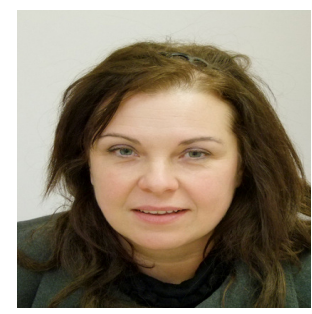

